\title{
USO DO CARBONATO DE SÓDIO COMO ALTERNATIVA PARA O PRÉ-TRATAMENTO DO BAGAÇO DE CANA-DE- AÇÚCAR SOB CONDIÇÕES AMENAS DE PRESSÃO E TEMPERATURA
}

Isadora C. V. Rodrigues; Paula J. Esteves, Walter Carvalho; Silvio S. Silva; Júlio C. dos Santos*

Escola de Engenharia de Lorena, Universidade de São Paulo, Departamento de Biotecnologia

E-mail para contato: jsant200@usp.br

\begin{abstract}
RESUMO - O aumento da digestibilidade enzimática de materiais como o bagaço de cana-de-açúcar tem sido considerado fundamental para processos visando à obtenção de etanol de segunda geração no Brasil. Neste trabalho, foi avaliado o uso do $\mathrm{Na}_{2} \mathrm{CO}_{3}$ para o pré-tratamento do bagaço visando ao aumento de sua digestibilidade por preparação comercial de celulases. Os pré-tratamentos foram feitos a $60^{\circ} \mathrm{C}$ sob pressão atmosférica, usando diferentes concentrações de $\mathrm{Na}_{2} \mathrm{CO}_{3}$ e razões mássicas bagaço:meio reacional, sendo a hidrólise enzimática realizada a $50^{\circ} \mathrm{C}$ por $24 \mathrm{~h}$. Os resultados obtidos mostraram que, nas condições estudadas, houve solubilização de cerca de $20-30 \%$ da hemicelulose do material original, sendo que, usando as condições mais severas de razão mássica álcali:bagaço empregadas, houve remoção de cerca de $30 \%$ tanto da hemicelulose quanto da lignina. Uma relação linear entre a razão mássica álcali:bagaço empregada no prétratamento e o rendimento de hidrólise da celulose na etapa enzimática foi observada. $\mathrm{O}$ uso de razão mássica $\mathrm{Na}_{2} \mathrm{CO}_{3}$ /bagaço de 1,2 triplicou o percentual de hidrólise da celulose (30\%) em comparação ao obtido com bagaço não tratado (10\%). Foi então demonstrado o potencial de aplicação de $\mathrm{Na}_{2} \mathrm{CO}_{3}$ no processo, sendo esta uma opção de pré-tratamento alcalino alternativa ao uso de $\mathrm{NaOH}$.
\end{abstract}

\section{INTRODUÇÃO}

O aproveitamento de materiais lignocelulósicos, os quais são os principais constituintes da biomassa vegetal de resíduos e sub-produtos agrícolas e florestais, tem sido objeto de pesquisas em várias partes do mundo visando-se à obtenção de produtos de interesse econômico e social (Kuhad e Singh, 1993; Cherubini, 2010). Para uso como matéria-prima em processos biotecnológicos, geralmente estes materiais precisam ser submetidos previamente a um processo capaz de transformar suas macromoléculas constituintes em compostos que possam ser metabolizados por microorganismos ou utilizados como substrato em processos enzimáticos. Um dos produtos que podem ser obtidos por via microbiológica a partir de lignocelulósicos é o etanol combustível. Processos para 


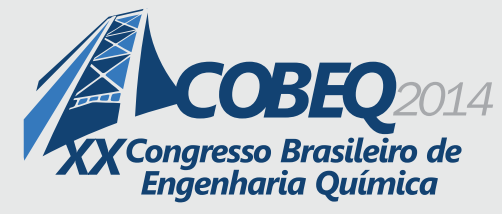

19 a 22 de outubro de 2014

Florianópolis/SC

obtenção deste composto a partir de biomassa vegetal são chamados "de segunda geração" e a busca de viabilização de sua produção tem sido intensa, principalmente tendo em vista a desejável substituição do uso de combustíveis de origem fóssil pelos de fonte renovável (Cherubini, 2010; Boudet, 2011).

O etanol pode ser obtido a partir da fermentação da glicose oriunda da fração macromolecular "celulose", principal constituinte da parede celular da biomassa vegetal. Para este fim, a celulose precisa ser hidrolisada e os trabalhos atuais têm sido direcionados à viabilização da hidrólise enzimática desta fração do material. Para que a hidrólise enzimática ocorra, no entanto, é necessário um pré-tratamento que modifique sua composição e a estrutura fechada formada pela celulose, hemicelulose e lignina, favorecendo o acesso dos biocatalisadores ao substrato (Agbor et al., 2011; Galbe e Zacchi, 2012). Este pré-tratamento tem sido feito principalmente pelo uso de ácidos diluídos em um processo sob condições de elevada pressão e temperatura (Alvira et al., 2010).

Uma alternativa proposta na literatura é referente ao uso de pré-tratamento alcalino, o qual pode ser feito sob condições mais brandas (Wu et al., 2011a; Wu et al., 2011b; Modenbach e Nokes, 2012). Entre os reagentes alcalinos mais empregados, destaca-se o hidróxido de sódio, embora outros álcalis, de menor custo, possuam potencial para uso no processo. Neste contexto, foram realizados experimentos para a avaliação do efeito do uso de carbonato de sódio no pré-tratamento do bagaço de cana-de-açúcar, matéria-prima escolhida pela sua abundância no Brasil.

\section{MATERIAIS E MÉTODOS}

\subsection{Preparo da Matéria Prima}

O bagaço de cana-de-açúcar foi adquirido da Usina São Francisco, Sertãozinho - SP. Secou-se o material ao sol até que fosse atingida umidade aproximada de $10 \%$, conforme medido em balança de infravermelho Mark M163 (BEL Engineering, Piracicaba, SP), para evitar possível contaminação microbiana durante a estocagem no laboratório. Após a secagem, o bagaço foi moído em um moinho de martelo marca Benedetti, modelo dupla 270 (Moinho Benedetti Ltda., Pinhal - SP), até que pelo menos $90 \%$ das partículas passassem por uma peneira padrão Tyler $10 \mathrm{MESH}$ (abertura 1,65 mm). O bagaço seco e moído foi caracterizado com relação a sua composição em extrativos, conforme descrito por Masarin et al. (2011), e em celulose, hemicelulose, lignina e cinzas, de acordo com metodologia descrita por Sluiter et al. (2014), sendo então empregado nos experimentos de prétratamento.

\subsection{Pré-Tratamento Da Biomassa}

Os experimentos foram realizados em frascos Erlenmeyer de $250 \mathrm{~mL}$ contendo um total de $100 \mathrm{~g}$ de meio reacional composto por bagaço e solução de carbonato de sódio, a temperatura constante de $60^{\circ} \mathrm{C}$ em uma banho com agitação (Lindberd/Blue M, SWB1122C-1, Waterbath, Asheville, Estados Unidos) de $110 \mathrm{rpm}$ durante 24h. Em seguida, procedeu-se à filtração a vácuo, sendo que o sólido 


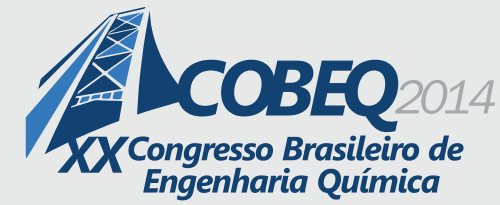

obtido foi separado para hidrolise enzimática e caracterização com relação a sua composição em celulose, hemicelulose e lignina de acordo com metodologia descrita por Sluiter et al. (2014). As reações foram feitas em triplicata, sendo empregados valores de concentração de $\mathrm{Na}_{2} \mathrm{CO}_{3}$ de $0,5 \mathrm{M}$ e $1 \mathrm{M}$ e razão mássica bagaço:meio reacional (massa seca) de $2,5 \%$ e 7,5\%, correspondendo a uma razão mássica $\mathrm{Na}_{2} \mathrm{CO}_{3}$ :bagaço de 0,2 a 1,2. A massa seca de bagaço recuperado após o procedimento de pré-tratamento foi medida após secagem em estufa a $105^{\circ} \mathrm{C}$. Com este valor, foi possível o cálculo da recuperação de cada uma das frações do material após o processo. Esta foi definida como a razão entre a massa seca de uma dada fração macromolecular do bagaço ao final do pré-tratamento e a massa seca inicial.

\subsection{Hidrólise Enzimática}

A hidrolise enzimática foi realizada em frascos Erlenmeyer de $50 \mathrm{~mL}$ com $15 \mathrm{~mL}$ de meio reacional contendo $2 \%$ de bagaço de cana de açúcar pré-tratado (massa seca), tampão de citrato de sódio $0,05 \mathrm{M} \mathrm{pH}=4,8$ e $10 \mathrm{FPU}$ enzima/g de bagaço, utilizando-se coquetel enzimático comercial Dyadic ${ }^{\circledR}$ Cellulase CP CONC (Dyadic International Inc., Jupiter, Flórida, USA). Os experimentos foram realizados em incubadora de movimento rotatório Quimis Q816M20 (Quimis Aparelhos Científicos, Diadema, SP, Brasil) com agitação de $150 \mathrm{rpm}$ durante $24 \mathrm{~h}$ a $50^{\circ} \mathrm{C}$. A concentração de glicose liberada pela hidrólise enzimática foi determinada por cromatografia liquida de alta eficiência (CLAE) empregando coluna BIO RAD AMINEX HPX 87p (300x7,8 mm), conforme Medina (2013).

\subsection{Expressão Dos Resultados}

Os experimentos/análises foram realizados em triplicata e os resultados expressos como valor médio \pm desvio padrão.

\section{RESULTADOS E DISCUSSÕES}

Inicialmente, fez-se uma caracterização da matéria-prima, a qual apresentou a seguinte

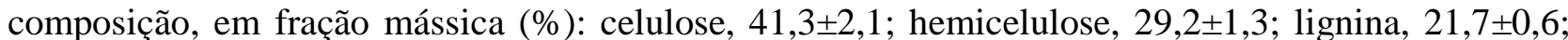
extrativos, 4,5 $\pm 0,4$; cinzas, $3,7 \pm 0,2$. Este material foi submetido ao pré-tratamento com carbonato de sódio, sendo a composição do sólido pré-tratado obtido mostrado na Tabela 1. Ainda com relação ao procedimento de pré-tratamento, a Figura 1 apresenta os valores de recuperação de cada uma das frações do material no procedimento de pré-tratamento.

Como pode ser observado, o material pré-tratado apresentou composição similar à obtida para a matéria-prima, sendo verificado, em quase todos os experimentos, um menor valor percentual de hemicelulose. Realmente, a observação dos dados da Figura 1 mostra que houve remoção desta fração carboidrato do bagaço em todas as condições avaliadas. A análise desta figura mostra ainda que, de uma forma geral, houve perda de 10-20\% de celulose, além remoção de 20-30\% de hemicelulose e de $0-30 \%$ de lignina. 
Tabela 1 - Caracterização composicional do bagaço de cana-de-açúcar pré-tratado com carbonato de sódio sob diferentes condições

\begin{tabular}{ccccccc}
\hline & \multicolumn{3}{c}{ Condições } & \multicolumn{3}{c}{ Composição (\%) } \\
\hline Experimento & $\begin{array}{c}\text { Concentração razão mássica } \\
\text { de } \mathbf{N a}_{\mathbf{2}} \mathbf{C O}_{\mathbf{3}} \\
(\mathbf{M})\end{array}$ & $\begin{array}{c}\text { bagaço:meio } \\
\text { beacional }\end{array}$ & $\begin{array}{c}\text { razão mássica } \\
\mathbf{N a}_{2} \mathbf{C O}_{3} \text { :bagaço }\end{array}$ & Celulose & Hemicelulose & Lignina \\
\hline 1 & 0,5 & 2,5 & 0,6 & $46,84 \pm 1,13$ & $28,18 \pm 1,68$ & $21,18 \pm 1,27$ \\
2 & 0,5 & 7,5 & 0,2 & $44,75 \pm 1,99$ & $25,80 \pm 0,33$ & $25,81 \pm 1,86$ \\
3 & 1,0 & 2,5 & 1,2 & $46,74 \pm 1,98$ & $25,77 \pm 0,71$ & $19,47 \pm 0,16$ \\
4 & 1,0 & 7,5 & 0,4 & $45,09 \pm 0,88$ & $25,63 \pm 1,36$ & $23,70 \pm 0,37$ \\
\hline
\end{tabular}

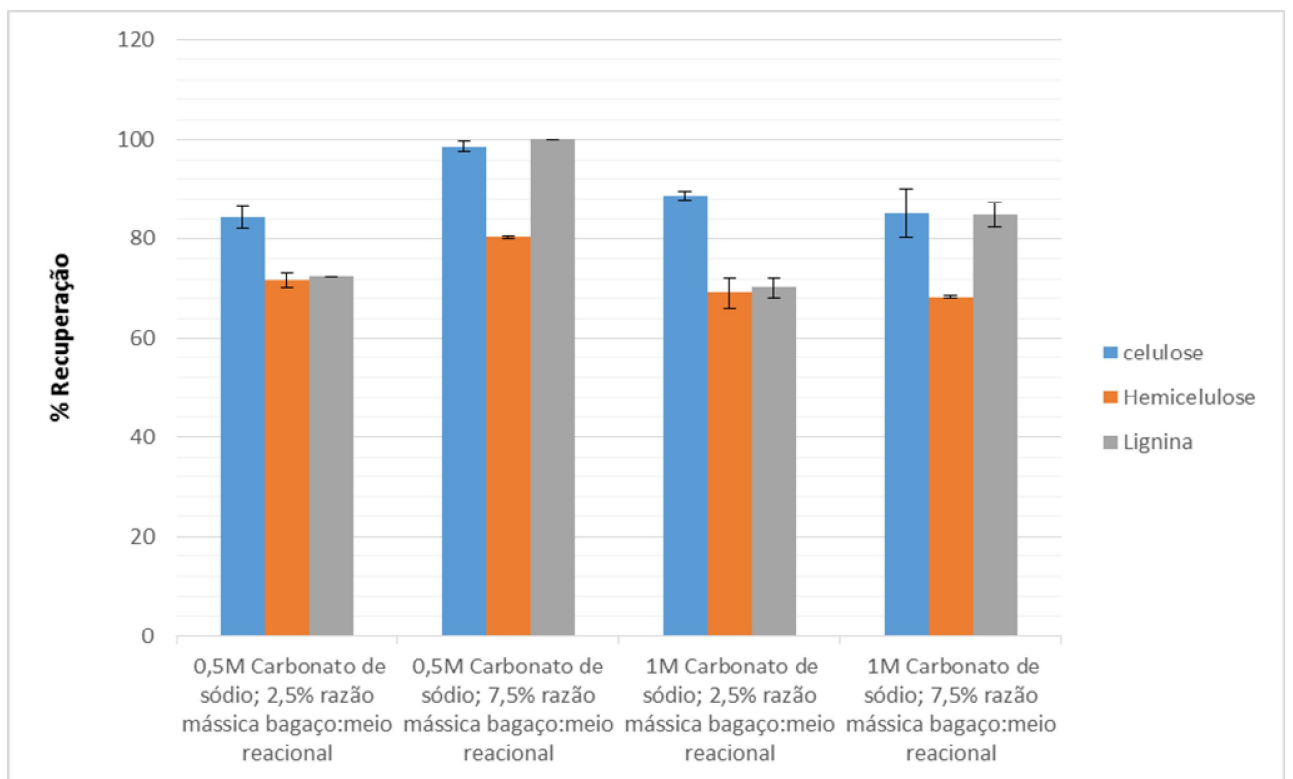

Figura 1 - Recuperação de celulose, hemicelulose e lignina no processo de pré-tratamento de bagaço de cana de açúcar com carbonato de sódio sob diferentes condições de concentração de $\mathrm{Na}_{2} \mathrm{CO}_{3}$ e razão mássica de bagaço no meio reacional

A hemicelulose foi, desta forma, a fração macromolecular do material mais removida no prétratamento com $\mathrm{Na}_{2} \mathrm{CO}_{3}$. De fato, embora processos alcalinos sejam usados em pré-tratamento da biomassa principalmente devido a sua capacidade de solubilização da lignina (Carvalheiro et al., 2008; Alvira et al., 2010), seu efeito na solubilização da hemicelulose é conhecido e usado com sucesso na extração desta fração do material. No presente trabalho, mesmo empregando as condições mais brandas em termos de razão mássica álcali:bagaço (experimento 2, Tabela 1), as quais praticamente não resultaram em remoção de celulose ou lignina do bagaço, cerca de $20 \%$ da hemicelulose foi solubilizada. Quando foram empregadas condições mais severas (experimento 3, 


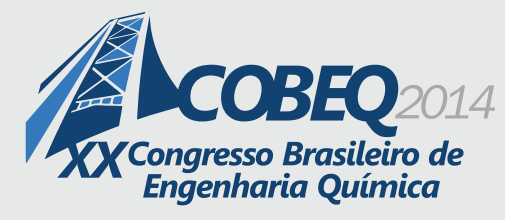

19 a 22 de outubro de 2014

Florianópolis/SC

Tabela 1), o percentual de lignina removido foi similar ao da hemicelulose - cerca de $30 \%$. Mesmo nesta condição, no entanto, a perda da celulose foi baixa, sendo possível recuperação de cerca de $90 \%$ desta após o processo.

$\mathrm{O}$ uso de maior relação $\mathrm{Na}_{2} \mathrm{CO}_{3}$ : bagaço no pré-tratamento favoreceu uma maior digestibilidade do material por preparação comercial de celulose na etapa subsequente de hidrólise enzimática (exp. 1 e 3, Tabela 2). A análise dos dados da Tabela 2 mostra que a relação entre a concentração de álcali e de bagaço foi o fator mais importante, ao invés da concentração de $\mathrm{Na}_{2} \mathrm{CO}_{3}$ e quantidade de sólidos presentes no meio, propriamente dito. Como mostrado na Figura 2, houve relação linear entre a razão álcali:bagaço no pré-tratamento e o rendimento de hidrólise.

Tabela2 -Digestibilidade enzimática do bagaço de cana-de-açúcar não tratado ou submetido ao prétratamento alcalino sob diferentes condições de concentração de álcalis e razão mássica bagaço:meio

\begin{tabular}{|c|c|c|}
\hline$\overline{\text { Experimento }}$ & Sistema/Condições & $\begin{array}{c}\text { Rendimento de hidrólise da celulose } \\
(\%)\end{array}$ \\
\hline \multirow[b]{2}{*}{1} & Bagaço não tratado & $11,00 \pm 2,40$ \\
\hline & Bagaço pré-tratado/ $\mathrm{Na}_{2} \mathrm{CO}_{3} 0,5 \mathrm{M} /$ & \\
\hline & razão mássica bagaço:meio reacional 2,5 & $20,45 \pm 2,10$ \\
\hline \multirow[t]{2}{*}{2} & Bagaço pré-tratado/ $\mathrm{Na}_{2} \mathrm{CO}_{3} 0,5 \mathrm{M} /$ razão & \\
\hline & mássica bagaço:meio reacional 7,5 & $14,21 \pm 0,70$ \\
\hline \multirow[t]{2}{*}{3} & Bagaço pré-tratado/ $\mathrm{Na}_{2} \mathrm{CO}_{3} 1,0 \mathrm{M} /$ razão & \\
\hline & mássica bagaço:meio reacional 2,5 & $31,05 \pm 1,45$ \\
\hline \multirow[t]{2}{*}{4} & Bagaço pré-tratado/ $\mathrm{Na}_{2} \mathrm{CO}_{3}$ 1,0 M / & \\
\hline & razão mássica bagaço:meio reacional 7,5 & $18,34 \pm 1,87$ \\
\hline
\end{tabular}

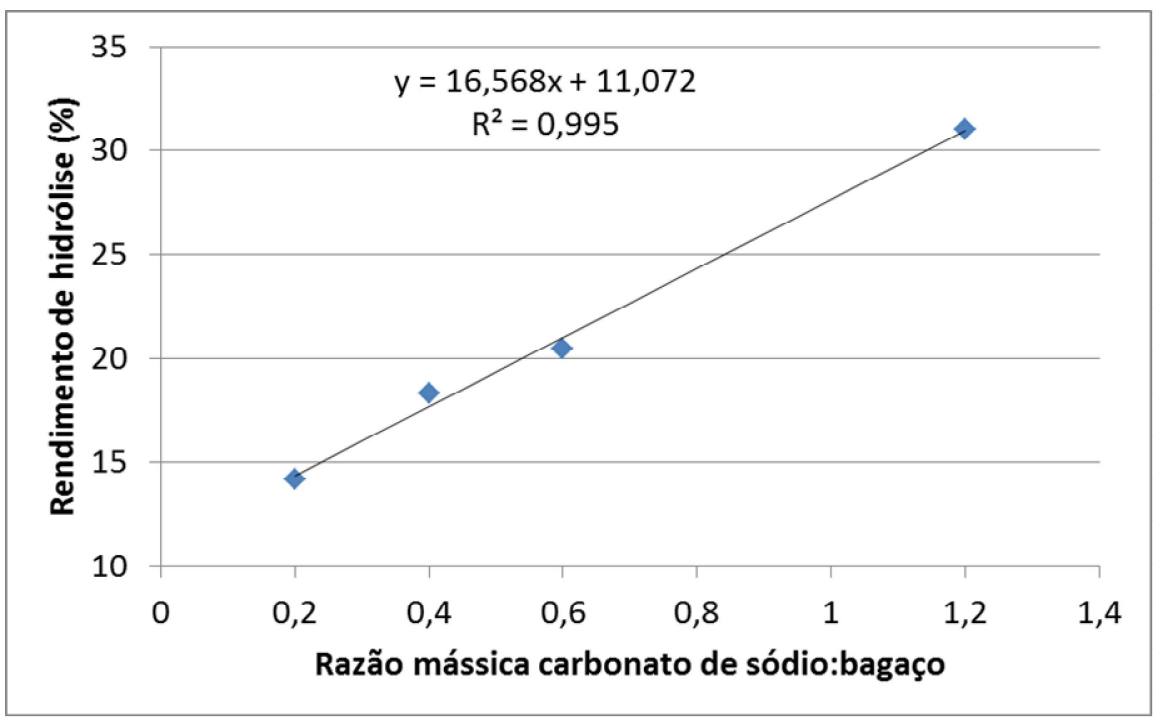

Figura 2 - Rendimento de hidrólise em função da razão mássica entre carbonato de sódio e bagaço empregada no pré-tratamento do bagaço de cana-de-açúcar 
$\mathrm{O}$ ajuste linear dos dados apresentados na Figura 2 indicam que, nas condições avaliadas, o aumento de um décimo na razão mássica $\mathrm{Na}_{2} \mathrm{CO}_{3}$ :bagaço no pré-tratamento resulta em aumento de 1,7 unidades percentuais no rendimento de hidrólise após $24 \mathrm{~h}$ de reação enzimática com $10 \mathrm{FPU} / \mathrm{g}$ de bagaço da preparação comercial avaliada.

\section{CONCLUSÃO}

O uso de carbonato de sódio no pré-tratamento alcalino do bagaço de cana-de-açúcar resultou, nas condições estudadas, em solubilização de cerca de 20-30\% da hemicelulose do material, sendo que, nas condições mais severas de razão mássica álcali:bagaço empregadas, houve remoção de cerca de $30 \%$ tanto da hemicelulose quanto da lignina. Nesta condição, cerca de $90 \%$ da celulose foi recuperada no material pré-tratado. Pôde-se obter um rendimento de hidrólise da celulose (representativo da digestibilidade enzimática do material) de $31 \%$ quando as condições foram $1 \mathrm{M}$ de $\mathrm{Na}_{2} \mathrm{CO}_{3}$ e 2,5\% de bagaço no meio, resultado cerca de 3 vezes superior em comparação ao material não tratado. Uma relação linear entre a razão mássica álcali:bagaço empregada no pré-tratamento e o rendimento de hidrólise da celulose foi observada. Embora experimentos de otimização de condições devam ser realizados tanto no pré-tratamento quanto na etapa de hidrólise, foi demonstrado o potencial de uso de carbonato de sódio no pré-tramento sob condições relativamente brandas de processo.

\section{REFERÊNCIAS}

AGBOR, V. B.; CICEK, N.; SPARLING, R.; BERLIN, A.; LEVIN, D. B. Biomass pretreatment: Fundamentals toward application. Biotechnology Advances, 29, 675-685, 2011.

ALVIRA, P.; TOMÁS-PEJÓ, E.; BALLESTEROS, M.; NEGRO, M. J. Pretreatment technologies for an efficient bioethanol production process based on enzymatic hydrolysis: A review. Bioresource Technology, 101, 4581-4861, 2010.

BOUDET, A. M. Editorial: A new era for lignocellulosics utilization through biotechnology. Comptes Rendus Biologies, 334, 777-780, 2011.

CARVALHEIRO F., DUARTE L. C.; GÍRIO F.M.: Hemicellulose biorefineries: a review on biomass pretreatments. Journal of Scientific \& Industrial Research, 67, 849-864, 2008.

CHERUBINI, F. The biorefinery concept: Using biomass instead of oil for producing energy and chemicals. Energy Conversion and Management, 51, 1412-1421, 2010. 
GALBE, M.; ZACCHI, G. Pretreatment: The key to efficient utilization of lignocellulosic materials. Biomass and bioenergy, 46, 70-78, 2012.

KUHAD, R. C., SINGH, A. Lignocellulose Biotechnology: Current and Future Prospects. Critical Reviews in Biotechnology, 13, 151-73, 1993.

MASARIN et al., Chemical composition and enzymatic digestibility of sugarcane clones selected for varied lignin content, Biotechnology for biofuels 4.55, 2011

MEDINA, K. J. D. . Produção de bioetanol a partir de hidrolisado hemicelulósico de bagaço de cana-de-açúcar empregando as leveduras Scheffersomyces (Pichia) stipitis NRRL Y-7124 e Candida shehatae UFMG HM 52.2 visando à aplicação em bioprocessos com campo eletromagnético. 2013. p.172, Lorena/SP: Universidade de São Paulo, Escola de Engenharia de Lorena, 2013, (Tese de Doutorado)

MODENBACH, A. A.; NOKES, E. S., The Use of High-Solids Loadings in Biomass Pretreatment - A Review, 6, 2012

SLUITER, A.; HAMES, B.; RUIZ, R. SCARLATA, C.; SLUITER, J. TEMPLETON, D.; CROCKER, D. Determination of structural carbohydrates and lignin in biomass, Natinal Renewable Energy Laboratory, Golden, CO., 2011. Disponível na internet em: http://www.nrel.gov/biomass/pdfs/42618.pdf. Consulta em: 21 de janeiro de 2014.

WU, L.; ARAKANE, M.; IKE, M.; WADA, M.; TAKAI, T.; GAU, M.; TOKUYASU, K. Low temperature alkali pretreatment for improving enzymatic digestibility of sweet sorghum bagasse for ethanol production. Bioresource Technology, 102, 4793-4799, 2011 a.

WU, L.; Li, Y.; ARAKANE, M.; IKE, M.; WADA, M.; TERAJIMA, Y.; ISHIKAWA, S.; TOKUYASU, K. Efficient conversion of sugarcane stalks into ethanol employing low temperature alkali pretreatment method. Bioresource Technology, 102, 11183-11188, $2011 b$ 